\title{
The psychological impact of the unpredictability of multiple sclerosis: a qualitative literature meta-synthesis
}

\begin{abstract}
Background: Multiple sclerosis (MS) is a neurological condition characterised by an unpredictable nature. This characteristic is reported to be the largest challenge for individuals with the condition. Chronic illness literature highlights the psychological impact of unpredictability, but there is a paucity of research examining this concept in MS.

Objectives: This systematic review aimed to explore the psychological impact of unpredictability in MS.

Methods: A meta-synthesis was conducted. A total of 6 studies with 59 participants were reviewed and critically appraised. Themes relating to the psychological impact of unpredictability were extracted and synthesised. Results: A preliminary model of the unpredictability of MS was developed highlighting the different psychological impacts of diagnosis, remission and relapse.

Conclusions: The psychological impact of unpredictability alters through diagnosis, remission and relapse. Further research is required to explore the experience of unpredictability in MS.
\end{abstract}

Key Words Multiple sclerosis, psychological impact, unpredictability, systematic review, qualitative, synthesis

Authors Hannah Rose Wilkinson is Trainee Clinical Psychologist, Trent Doctorate in Clinical Psychology, Faculty of Humanities, Languages and Social Sciences, University of Lincoln; Roshan das Nair is Research Tutor and Consultant Clinical Psychologist, Trent Doctorate in Clinical Psychology, Institute of Work, Health and Organisations, University of Nottingham

Correspondence Hannah.wilkinson5@nhs.net

Accepted 19 June 2013

This article has been subject to double-blind peer review.

\section{Hannah Rose Wilkinson and Roshan das Nair}

Multiple sclerosis (MS) is a condition of the central nervous system affecting both the brain and the spinal cord and resulting in a variety of symptoms. It affects around 100000 people in the UK and has no known cure (Multiple Sclerosis Society, 2008). Individuals with MS experience irregular relapses and a downward progression of the illness, and the unknown eventual level of functional impairment and disruptions in daily functioning contribute to the perception of it being a highly unpredictable illness (Mullins et al, 2001; Thomas et al, 2006).
Wilkinson, H., \& dasNair, R.* (2013). The Psychological Impact of Living with an Unpredictable Illness: A MetaSynthesis of Qualitative Research Literature in Multiple Sclerosis. British Journal of Neuroscience Nursing, 9(4), 172-178. 
Research has emphasised the impact of MS, highlighting the emotional and physical effects on theindividual and their family and friends (Aronson, 1997; Cheung and Hocking, 2004; Mutch, 2010). A standardised measure of the impact of MS includes both physical and psychological factors. The nine psychological effects measured are: feeling unwell, sleep problems, fatigue, MS-related worries, anxiety, irritability, concentration problems, lowered selfconfidence, and depression (Hobart et al, 2001). These effects are suggested to cause functional deficits resulting in reduced quality of life.

The UK Department of Health (DH, 1992) has highlighted the importance of exploring the lived experience of people with a chronic illness to enable services to improve quality of life. Consequently, interest has grown in qualitative studies exploring the lived experience of those with MS and their carers (Miller, 1997; Koopman and Schweitzer, 1999; Cheung and Hocking, 2004; Shevil and Finlayson, 2006; Malcomson et al, 2008; Olsson et al, 2008; Mutch, 2010; Moriya and Suzuki, 2011; Vick, 2013). Unpredictability is one of the largest challenges in MS (Malcomson et al, 2008). However, despite a wealth of research exploring the effects of uncertainty and unpredictability in chronic illnesses (McCormick, 2002; Thorne et al, 2002), the impact of unpredictability in MS has not been extensively examined.

This review aims to systematically explore the psychological impact of living with unpredictability in MS, drawing on qualitative research. Compared with quantitative research, qualitative research enables researchers to get closer to patients' experiences, by exploring not only what is being said but also how it is said. A nuanced understanding of the psychological impact of unpredictability could offer guidance for future research and identify areas of importance to services and future practice.

\section{Methods}

\section{Systematic literature search}

Inclusion and exclusion criteria were defined a priori. Papers were considered if they included participants with MS (PwMS) and explored their experience of living with MS, were primary research, discussed the psychological impact of the unpredictability of MS, used qualitative methods of data collection and analysis, and were in the English language. Papers using 
mixed methods were also considered if there was a clear qualitative component and themes could be clearly extracted. The search included papers published up until the point of analysis.

Psychological impact was defined as any cognitive (e.g. problems with memory), behavioural (e.g. feeling tired) or affective (e.g. feeling depressed) changes that an individual perceived to result from the unpredictability of their MS. Unpredictability is defined as an inability to foretell the future (McCormick, 2002). By definition, unpredictability is a factor of uncertainty (McCormick, 2002; Neville, 2003). The concepts are theoretically distinguishable but there is considerable overlap and inconsistency in the use and definition of these terms in the literature. Therefore, for this review uncertainty and unpredictability were considered synonymous. Unpredictability was defined as participants' perception of changeableness or uncertainty of their symptoms, diagnosis and relapses. It also included unpredictability/uncertainty encountered in day-to-day living with MS.

\section{Search terms}

Search terms were developed by HRW in consultation with RdN. The terms used were:

- 1. '*Multiple sclerosis'

2. 'Qualitative*' OR 'lived experience' OR 'patient perspective' OR 'experience'

- 3. 'Psycholog*' OR 'psychological impact' OR 'psychosocial aspects of illness' OR 'psychological adaptation' OR 'attitude to illness' OR 'disease progression' OR 'anxiet*' OR 'depress*' OR 'coping' OR 'cogniti*' OR 'behaviou*' OR 'emotio*' OR 'adjustment'

- 4. 'Psycholog*' OR 'psychological impact' OR 'adjustment' OR 'coping' OR 'anxiety' OR 'depression' OR 'emotion*'

- 5. 'unpredict*' OR 'uncertain*'

- 6. 4 AND 5

7. 6 OR 3

\section{1 AND 2 AND 7.}

A systematic search was conducted through the NHS Evidence search engine. The AMED, EMBASE, Medline, PsychINFO, British Nursing Index (BNI) and CINAHL electronic bibliographic databases were searched in July 2012. The databases represented the literature from medical, nursing and social science disciplines. Alerts through NHS Evidence were set up to indicate new relevant studies during the review period (1 July-6 December 2012). A separate search was conducted on Google Scholar using the key terms 'qualitative', 'multiple sclerosis' and 'psychological impact' on 16 July 2012, and the first 100 citations were checked for relevance. Finally, the reference lists of all included papers provided an additional search strategy. Each citation was checked for relevance using the title; where relevance was in doubt the abstract was obtained. In cases where the abstract was 
unavailable or insufficient information was provided to ascertain relevance, the full text was obtained.

\section{Meta-synthesis}

A meta-synthesis was conducted from a critical realist epistemological position, acknowledging that data can provide insight into reality and that environmental factors may affect an individual's experiences (Harper, 2012). It is recognised that the reviewed studies offer a secondarylevel interpretation of the primary data collected from the participants and that interpretations are influenced by the researchers' own lived experiences, situation and epistemological stance. A meta-synthesis of the secondary-level interpretation brings together the methodological and contextual complexities and provides a third level of interpretation along with its own epistemological slant (Zimmer, 2006).

There is no consensus regarding the most appropriate method for meta-synthesis. However, Walsh and Downe (2005) outlined the generally accepted techniques and stages involved, and these were used to guide the present review.

\section{Appraisal of studies}

Quality appraisals are widely debated among qualitative researchers. There is insufficient evidence to ascertain the most appropriate tool for, the rigour of or the added value of quality appraisals (Dixon-Woods et al, 2004; Noyes et al, 2008). The review considered studies with different methodologies and hence the Critical Appraisal Skills Programme tool (CASP, 2010) was used as it meets the criteria outlined by Dixon-Woods et al (2004) that are deemed useful in qualitative studies using different methodologies. The CASP was used to explore and interpret reviewed studies, as suggested by Noyes et al (2008). However, it was not used to discount studies, given that all studies provide information (Sherwood, 1997), and it is deemed inappropriate for qualitative reviews to use appraisal tools to make decisions about including or excluding a study (Dixon-Woods et al, 2004).

Therefore, any quality issues identified were considered in the synthesis of reviewed studies to reduce the impact and distortion on the interpretation of the study (Dixon-Woods et al, 2004).

All included studies were thoroughly examined by HRW, and general characteristics were extracted and tabulated. These included study aims, sample size, location, sample composition (including gender and age range), data collection method, data analysis method and key findings. These were crosschecked by $\mathrm{RdN}$ for reliability. Preservation of the original studies was maintained by reproducing the original text from included studies (Walsh and Downe, 2005).

\section{Reciprocal translation}

Themes relating to the psychological impact of unpredictability were extracted by HRW and discussed 


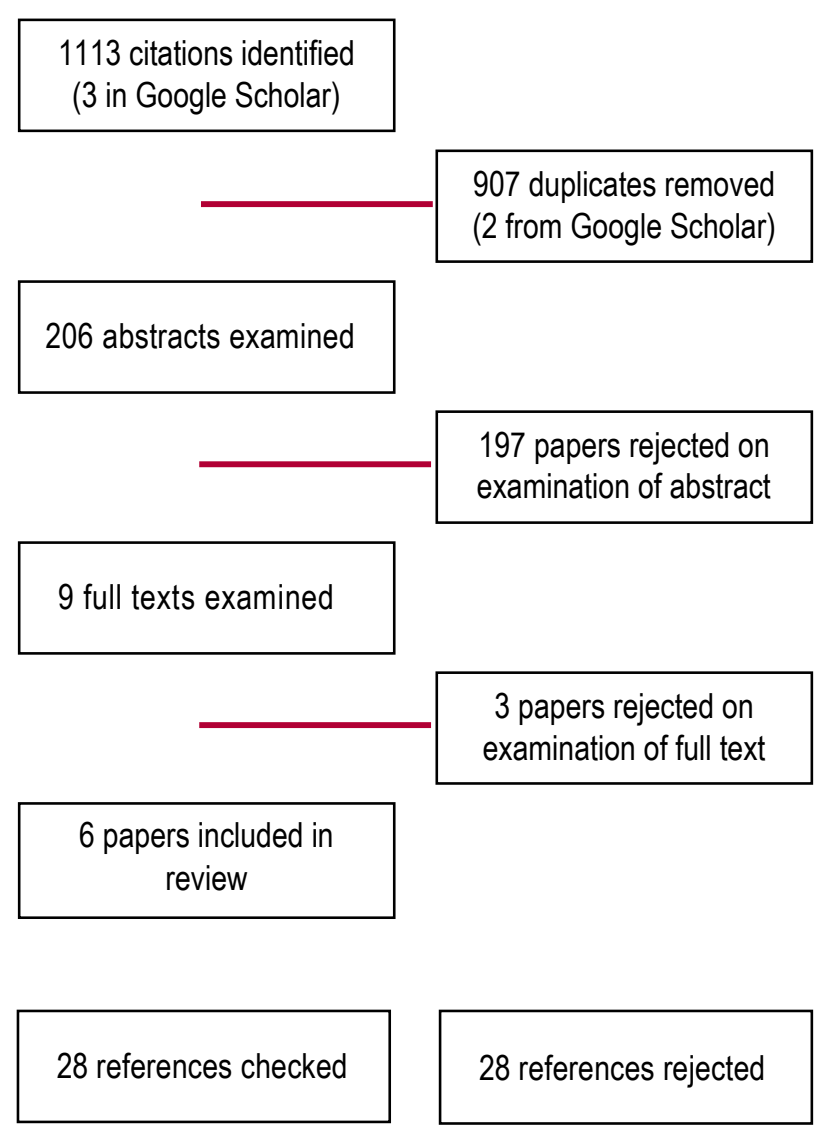

Figure 1. Search process and outcomes

with RdN. Themes were identified from the results sections of included papers to preserve the PwMS' perceptions. Themes were considered across the studies, identifying commonalities and dissonance, and were grouped together to develop concepts.

\section{Synthesis of translation}

The themes extracted were refined to develop a model of unpredictability in MS. This occurred through a process of returning to the original text to explore the origin of the theme, identifying how the theme related to unpredictability in each reviewed study, and comparing and contrasting across studies. For instance, a theme of perceived loss of control was identified in three studies. Commonalities and differences between these papers were explored, enabling an interpretation of the individual's experience of perceived loss of control due to the unpredictable nature of MS.

\section{Results}

\section{Literature search}

Figure 1 outlines the search process and outcomes. Of the 197 abstracts excluded, 14 did not include PwMS, 9 were not in English, 7 did not report the MS experience, 90 were quantitative, 25 were not primary research, and 52 did not explore the psychological impact. Of the three citations rejected after

examination of the full text, two made no reference to unpredictability (Courts et al, 2004; Yorkston et al, 2010) and one gave no indication of psychological effects of unpredictability (Kirkpatrick Pinson et al, 2009). Table 1 provides a summary of the six papers included in the review and assigns each a number that is used below and in the results section as a shorthand reference.

\section{Appraisal of studies}

All reviewed studies provided clear statements of the research aims and were deemed appropriate for an enquiry using qualitative methodology. Four studies reported making credibility checks (1,2,4 and 5) and saturation was reported in two studies ( 2 and 4 ). This highlights the rigour and reliability of the themes identified in these studies. Conversely, a majority of the studies did not state whether the relationship between the researchers and participants was considered, which is an important consideration in qualitative research. Epistemological stances were not stated and hence caution was exercised when extracting themes for synthesis.

Comparison of general study characteristics All six studies explored experiences of MS. Four focussed on the effects of symptoms of MS (1, 4, 5 and 6), but each study looked at different aspects of the experience. The studies were conducted between 1997 and 2011, and sample sizes ranged from 4 to 17 participants, with one study recruiting only female participants. The samples included individuals who had lived with MS for 6 months to 22 years. Two studies did not report their location (1 and 5); the others were conducted in Europe and North America. A majority of the studies did not report the ethnicity of the participants, therefore it is uncertain how culturally diverse these groups were.

Data were collected through individual interviews, except for one study (2) that used focus groups. Data analysis methods varied across studies and included thematic analysis (1 and 2), phenomenological hermeneutic interpretation (3,4 and 6) and constant comparison (5). The key findings of the studies included the effects of MS on the individual; the range of effects, from affective to physical symptoms; and the meanings applied by PwMS to enable successful coping. The key overarching theme identified was the effect of MS on an individual's daily life.

\section{Reciprocal translation}

Themes identifying the psychological impact of unpredictability were extracted from the reviewed studies. Cognitive, behavioural and affective impacts were identified. The main psychological impacts iden- 
tified were perceived loss, isolation/withdrawal, and emotional distress. Sub-themes were identified within 


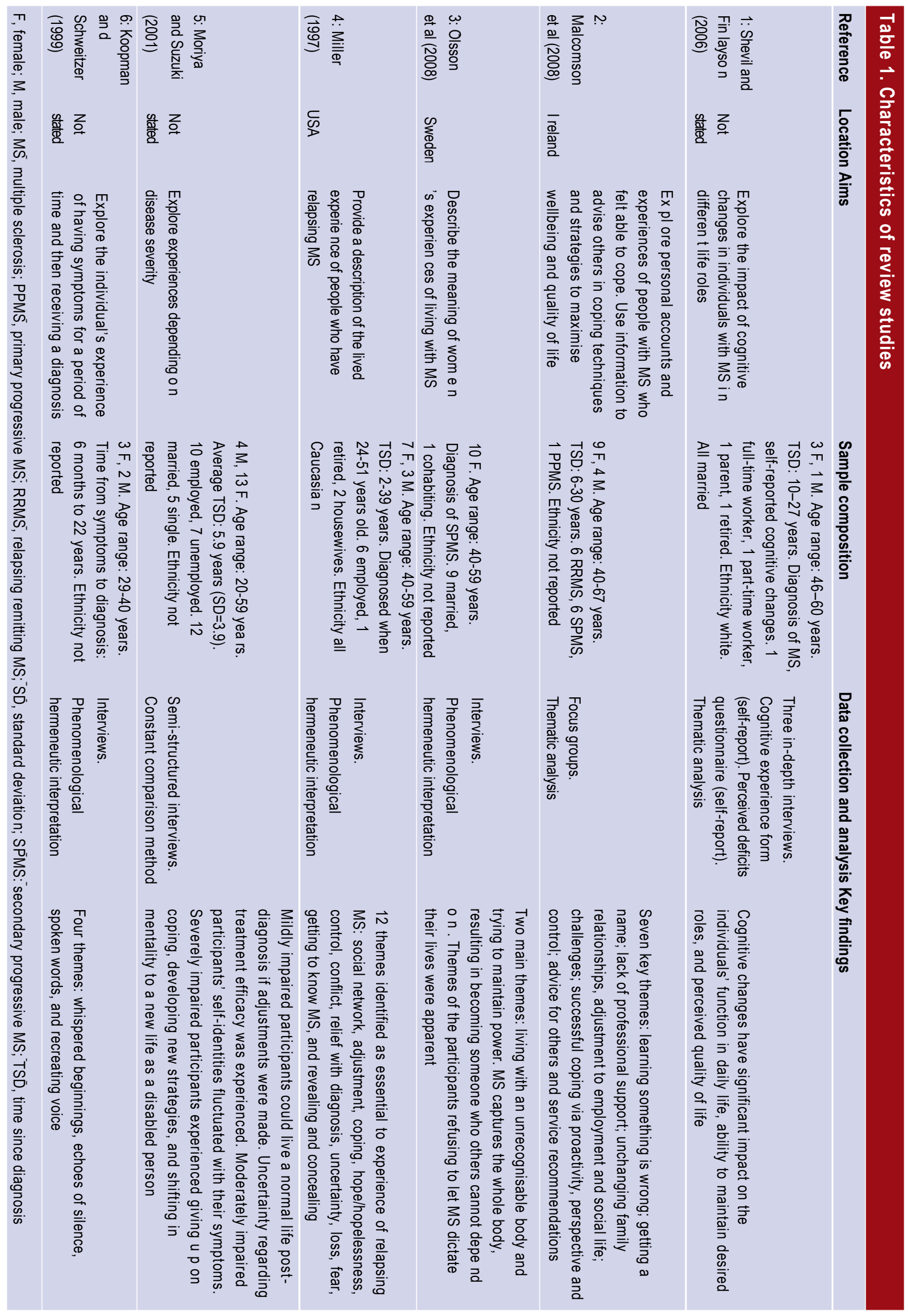




\begin{tabular}{|c|c|c|c|c|c|c|c|c|}
\hline & Perceived loss: & + & - & + & + & + & + & \\
\hline & - Control & + & - & + & & + & - & - \\
\hline & - Independence & + & - & - & & + & - & - \\
\hline & - Purpose & - & - & - & & - & + & - \\
\hline Cognitive & - Self-confidence & - & - & + & & - & - & - \\
\hline Behavioura & lation/withdrawal & & & & & & & -+ \\
\hline & Emotional distress: & + & + & + & + & + & - & \\
\hline & - Anxiety & - & + & - & & - & + & + \\
\hline & - Fear & - & + & + & & + & - & . \\
\hline Attectıve & - Anger/Frustration & + & - & - & & - & 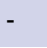 & 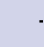 \\
\hline themes : & sub-themes & & & & & & & \\
\hline Domain & Themes and sub-themes 1 & & 2 & 3 & & 4 & 5 & 6 \\
\hline
\end{tabular}

these constructs. Table 2 summarises which of the papers were sources for these themes and subthemes.

\section{Feelings of loss}

A perception of loss was identified in five of the six papers. Three papers identified a loss of control due to the unpredictable nature of the symptoms (1,3 and 4). Unpredictable cognitive changes were described as a rollercoaster ride of temporal unpredictability owing to the timing of an inevitable event being unknown:

'... it's like you are on top of a hill and you know it is going to drop down.' $(1$, p785)

The rollercoaster ride led participants to feel that they were losing control (1). Unpredictable physical symptoms resulted in the participants no longer feeling in charge of their body, causing changes to their daily functioning (3). They could no longer trust their body and/or know whether they could complete plans that were important to them (3). One participant highlighted the most disturbing aspect of their MS as the unpredictable 'seizures', causing a sense of loss of control and resulting in emotional distress (4).

A perceived loss of independence due to the unpredictable symptoms was highlighted (1 and 4). This loss resulted in negative emotional reactions (1) such as a feeling of being broken, like a 'dripping faucet' (4, p299).

Those who experienced moderately severe MS perceived a loss of purpose due to their unclear future (5):
'If I had to try hard in order to get better, I would have a purpose. But just because I try hard, it doesn't mean I'll get better. I don't know what my goal should be.' $(5$, p598) 
Finally, a sense of loss of self-confidence in one's own ability was identified due to the unpredictable physical effects (3):

'You get insecure in yourself many times ... I try ... Can I manage this ... will I have enough strength to walk that distance ... insecure ... insecure ...

I have become in many ways.' $(3, \mathrm{p} 421)$

\section{Behavioural impact}

A behavioural impact of perceived social isolation was also reported (1, 3 and 6). A sense of loneliness was perceived prior to diagnosis, and the unpredictable symptoms and uncertain process of diagnosis also left participants lonely in their wait for responses to their investigations (6). The sense of unpredictability and unanswered questions resulted in 'being left wondering alone' (3; p 422). Isolation was further exacerbated by the symptoms of MS:

'[Cognitive changes] kind of closed down the parameters I live.' (1, p786)

The changes affected communication abilities:

'It's understanding the signals I'm getting from other people and a lot of the times I don't decipher it right.' $(1$, p785)

Participants removed themselves from these situations, resulting in isolation (1).

\section{Affective impacts}

Unpredictability created emotional distress, in particular anxiety $(2,5$, and 6). Anger and frustration (1) and fear (2, 3 and 4) were also identified. The emotional strains of unpredictable cognitive problems were referred to as a 'pain in the neck that drives me nuts' (1, p785).

Anxiety and fear were elicited by the unpredictability of the illness rather than the specific symptoms. A 'fear of the unknown' was apparent $(2,3$, and 4$)$ at two time points of the individual's MS journey: diagnosis and relapses. Individuals did not know what their level of function would be when they woke up each morning (4). The experience of relapses caused a fear of a loss of independence (2). Temporal unpredictability created a sense of anxiety in waiting for anticipated problems and interruptions to their lives (2, 5 and 6$)$.

\section{Synthesis of translation}

The cognitive, affective and behavioural impacts of unpredictability were apparent throughout the course of MS (Table 3). Diagnosis, remission and relapse have different forms of unpredictability and provoke different responses from patients. Temporal unpredictability, where a patient knows inevitable harm will 
occur but is unsure when, is identified during periods of remission. Event unpredictability, whereby the patient knows the timing of the event but does not know the extent of the problems to be encountered, occurs during diagnosis and relapse periods. The response to the different forms of unpredictability results in different cognitive, affective and behavioural responses. During periods of remission individuals experience day-to-day concerns, highlighting the impact of the cognitive and behavioural responses. The affective response turns from fear to anxiety, frustration and anger. Periods of diagnosis and relapse produce large affective responses, resulting in a sense of loneliness. Cognitive and affective impacts are not separate. Perceived loss of control and independence appeared to be related to the affective responses experienced.

Dissonance was identified between the individuals' perceptions of avoidance and the narrative of their behaviours. The narratives indicated that parameters of the participants' lives had altered and hence they may be unable to complete certain tasks. Avoidance was noted in those who experienced moderately severe MS and hence may indicate a mechanism of adapting to daily unpredictability.

\section{Discussion}

Unpredictability is a major challenge to PwMS (Malcomson et al, 2008), yet there is a paucity of research exploring their experiences of it. The present review provides a preliminary model explaining the psychological impact of unpredictability over the course of an individual's journey with MS.

In chronic relapsing illnesses, unpredictability has been suggested to relate to the prognosis of the illness, when symptoms will occur and whether there will be relapses (Mishel and Braden, 1988). The present review supports this claim. The psychological impact of unpredictability is continuous yet is dependent on the current experience (diagnosis, remission or relapse). The event unpredictability of diagnosis and relapse creates affective responses that result in a sense of loneliness. The temporal unpredictability of remission causes constant reappraisal of the self and has an impact on cognitive, affective and behavioural responses.

The identification of different forms of unpredictability and the different psychological responses supports claims made by Monat et al (1972). Event unpredictability creates an increased level of affective arousal, whereas temporal unpredictability leads to avoidance. An avoidant response develops as a form of cognitive coping (Monat et al, 1972).

Continual changes in functional ability threaten an individual's identity. Avoidance of situations that challenge one's identity may serve as a form of coping, reducing reappraisals of ability and identity. The narratives surrounding avoidant behaviour 
indicate that it is used to protect identity and beliefs around abilities, yet narrative dissonance indicates that people's awareness of their functional abilities does not correspond with their beliefs. The event unpredictability of a relapse creates fear that subsides during remission, mirroring the description of uncertainty (Mishel, 1990); however, temporal unpredictability maintains the affective response of anxiety. The individual's focus shifts from long-term goals to day-to-day matters (Neville, 2003).

\section{Implications for future research}

Research exploring event and temporal unpredictability could further inform services and future practice in relation to coping with and adjusting to MS.

Furthermore, daily fluctuations and the continual psychological distress of remission periods highlight the need for further research to explore the experience of unpredictability during these periods.

The present review was unable to reliably distinguish between unpredictability and uncertainty due to a lack of consensus in the definition. Uncertainty is reported to diminish over time as individuals become accustomed to the changes in their symptoms.

Adjustment to uncertainty and acceptance of one's new identity and limitations causes fear to subside (Charmaz, 1995; Miller, 1997). Miller (1997) reported that participants did not express strong negative feelings or a sense of loss of control due to uncertainty. However, the unpredictability creates continual distress to PwMS (Miller, 1997). Mishel (1990) stated that when uncertainty escalates, such as in a relapse, it exceeds tolerance levels and shifts the individual to a new way of life. Uncertainty can be viewed as a positive concept creating the opportunity to view life differently. The participants in the studies reviewed here did not articulate a positive attitude towards unpredictability, which may suggest a difference
Table 3. Model of the psychological impact of unpredictability in MS

\begin{tabular}{|c|c|c|c|}
\hline Stage & Cognitive & \multicolumn{2}{|c|}{ Affective Behavioural } \\
\hline \multirow[t]{3}{*}{$\begin{array}{l}\text { Diagnosis: } \\
\text { event } \\
\text { unpredictability } r\end{array}$} & $\begin{array}{l}\text { I'm not in control and I don't } \\
\text { know what's happening to } \\
\text { e (immediate losses) }\end{array}$ & Fear & $\begin{array}{l}\text { Inhibition and } \\
\text { withdrawal } \\
\text { Sense of loneliness }\end{array}$ \\
\hline & $\begin{array}{l}\text { I don't know when things will } \\
\text { get worse (loss of control) }\end{array}$ & Anxiety & Avoidance \\
\hline & $\begin{array}{l}\text { Can I do this task? } \\
\text { (Self-confidence) }\end{array}$ & Anxiety & Avoidance \\
\hline $\begin{array}{l}\text { Remission: } \\
\text { temporal } \\
\text { unpredictability }\end{array}$ & $\begin{array}{l}\text { I can't do what I used to (loss } \\
\text { of purpose and independence) }\end{array}$ & $\begin{array}{l}\text { Frustration/ } \\
\text { anger }\end{array}$ & $\begin{array}{l}\text { Desistance/'give } \\
\text { up' (behavioural } \\
\text { deactivation) }\end{array}$ \\
\hline \multicolumn{2}{|c|}{$\begin{array}{l}\text { Relapse: event know how bad it will be } \\
\text { unpredictability (immediate losses) }\end{array}$} & Fear & $\begin{array}{l}\text { Inhibition and } \\
\text { withdrawal }\end{array}$ \\
\hline
\end{tabular}




\section{KEY POINTS}

- Multiple sclerosis (MS) is characterised by an unpredictable nature.

- This characteristic is reported to be the largest challenge for individuals with the condition.

- The psychological impact of unpredictability alters through diagnosis, remission and relapse.

- Further research is required to explore the experience of unpredictability in MS.

between unpredictability and uncertainty. The discrepancy noted by Miller (1997) highlights the importance of distinguishing between these concepts and exploring them individually.

\section{Limitations}

The review was limited by the lack of research on the psychological impact of unpredictability. The reviewed studies drew samples only from Europe and North America, therefore generalisation of the results is difficult. The review examined studies that were methodologically different, but without knowledge of the epistemological stances of the studies it is likely that some content from the primary studies may have been lost (Zimmer, 2006).

\section{Conclusion}

The unpredictability of MS is a challenge that needs to be addressed in order to provide appropriate services, and clinicians need to be mindful of this. Increasing awareness of the impact of unpredictability that individuals face can help direct the services offered, tailoring them to the identified target times and areas. Unpredictability has been found to have impacts on individuals' cognition, affect and behaviours, hence practitioners need to address these areas. Further research into the psychological impacts of unpredictability in MS, such as longitudinal studies exploring the impact through individuals' journeys, could provide further insight and aid tailoring of services to an individual in specific times of need.

Aronson KJ (1997) Quality of life among persons with multiple sclerosis and their caregivers. Neurology 48(1): 74-80

Charmaz K (1995) The body, identity and self: adapting to impairment. Sociol $Q$ 36(4): 657-80

Cheung J, Hocking P (2004) Caring as worrying: the experience of spousal carers. J Adv Nurs 47(5): 475-82

Courts NF, Buchanan EM, Werstlein PO (2004) Focus groups:

the lived experience of participants with multiple sclerosis. $J$ Neurosci Nurs 36(1): 42-7

Critical Appraisal Skills Programme (2010) Making sense of evidence about clinical effectiveness: 10 questions to help you make sense of qualitative research. http://www.casp-uk.net/
wp-content/uploads/2011/11/CASP_Qualitative_Appraisal_ Checklist_14oct10.pdf (accessed 12 July 2013)

Department of Health (1992) Secretaries of State for Health Social Services, Scotland and Wales. Health of the Nation, London

Dixon-Woods M, Shaw RL, Agarwal S, Smith JA (2004) The problem of appraising qualitative research. Qual Saf Health Care 13(3): 223-225

Harper D (2012) Choosing a qualitative research method. In: Harper D, Thompson AR (eds) Qualitative Research Methods in Mental Health Psychotherapy: A Guide for Students and Practitioners. Wiley-Blackwell, UK: 83-98

Hobart J, Lamping D, Fitzpatrick R, Riazi A, Thompson A (2001) The Multiple Sclerosis Impact Scale (MSIS-29): a new patientbased outcome measure. Brain 124(5): 926-73

Koopman W, Schweitzer A (1999) The journey to multiple sclerosis: a qualitative study. J Neurosci Nurs 31(1): 17-26

Malcomson KS, Lowe-Strong AS, Dunwoody L (2008) What can we learn from the personal insights of individuals living and coping with multiple sclerosis? 30(9): 662-74

McCormick KM (2002) A concept analysis of uncertainty in illness. J Nurs Scholarsh 34(2): 127-31

Miller CM (1997) The lived experience of relapsing multiple sclerosis: a phenomenological study. J Neurosci Nurs 29(5): 294-304

Mishel MH (1990) Reconceptualization of the uncertainty in illness theory. Image J Nurs Sch 22(4): 256-62

Mishel MHH, Braden CJJ (1988) Finding meaning: antecedents of uncertainty in illness. Nurs Res 27(2): 98-127

Monat A, Averill JR, Lazarus RS (1972) Anticipatory stress and coping reactions under various conditions of uncertainty. J Pers Soc Psychol 24(2): 237-53

Moriya R, Suzuki S (2011) A qualitative study relating to the experiences of people with MS: differences by disease severity. Br J Neurosci Nurs 7(4): 593-600

Mullins LL, Cote MP, Fuemmeler BF, Jean VM, Beatty WW, Paul RH (2001) Illness intrusiveness, uncertainty, and distress in individuals with multiple sclerosis. Rehabil Psychol 46(2): 13953

Multiple Sclerosis Society (2008) What is MS? Information on diagnosis, symptoms, treatment, management and sources of support. www.mssociety.org.uk/sites/default/files/Documents/ Core pubs/What is MS English 0210 - web.pdf (accessed 12 July 2013)

Mutch K (2010) In sickness and in health: experience of caring for a spouse with MS. Br J Nurs 19(4): 214-9

Neville KL (2003) Uncertainty in illness. An integrative review. Orthop Nurs 22(3): 206-14

Noyes J, Popay J, Pearson A, Hannes K, Booth A (2008) Qualitative research and Cochrane reviews. In: Higgins JPT, Green S (eds) Cochrane Handbookfor Systematic Reviews of Interventions. John Wiley \& Sons, UK: 571-92

Olsson M, Lexell J, Söderberg S (2008) The meaning of women's experiences of living with multiple sclerosis. Health Care Women Int 29(4): 416-30

Kirkpatrick Pinson DM, Ottens AJ, Fisher TA (2009) Women coping successfully with multiple sclerosis and the precursors of change. Qual Health Res 19(2): 181-93

Sherwood G (1997) Meta-synthesis: merging qualitative studies to develop nursing knowledge. Int J Human Caring 3(1): 37-42

Shevil E, Finlayson M (2006) Perceptions of persons with multiple sclerosis on cognitive changes and their impact on daily life. Disabil Rehabil 28(12): 779-88

Thomas PW, Thomas S, Hillier C, Galvin K, Baker R (2006) Psychological interventions for multiple sclerosis. Cochrane Database Syst Rev 1: CD004431

Thorne S, Paterson B, Acorn S, Canam C, Joachim G, Jillings C (2002) Chronic illness experience: insights from a metastudy. Qual Health Res 12(4): 437-52

Vick A (2013) The embodied experience of episodic disability among women with multiple sclerosis. Disabil Soc 28(2): 176-89

Walsh D, Downe S (2005) Meta-synthesis method for qualitative research: a literature review. J Adv Nurs 50(2): 204-11

Yorkston KM, McMullan KA, Molton I, Jensen MP (2010) Pathways of change experienced by people aging with disability: a focus group study. Disabil Rehabil 32(20): 1697-704

Zimmer L (2006) Qualitative meta-synthesis: a question of dialoguing with texts. J Adv Nurs 53(3): 311-8 
